\section{FUNCTIONAL MAGNETIC RESONANCE IMAGING FOR AUTISM SPECTRUM DISORDER DETECTION USING DEEP LEARNING}

\author{
R. Nur Syahindah Husna, A. R. Syafeeza*, Norihan Abdul Hamid, Y. \\ C. Wong, R. Atikah Raihan
}

Machine Learning \& Signal Processing (MLSP) Research Group, Centre for Telecommunication Research \& Innovation (CeTRI), Fakulti Kejuruteraan Elektronik dan Kejuruteraan Komputer (FKEKK), Universiti Teknikal Malaysia Melaka Hang Tuah Jaya, 76100 Durian Tunggal, Melaka, Malaysia
Article history

Received

9 December 2020

Received in revised form

9 March 2021

Accepted

17 March 2021

Published online

22 April 2021

*Corresponding author syafeeza@utem.edu.my

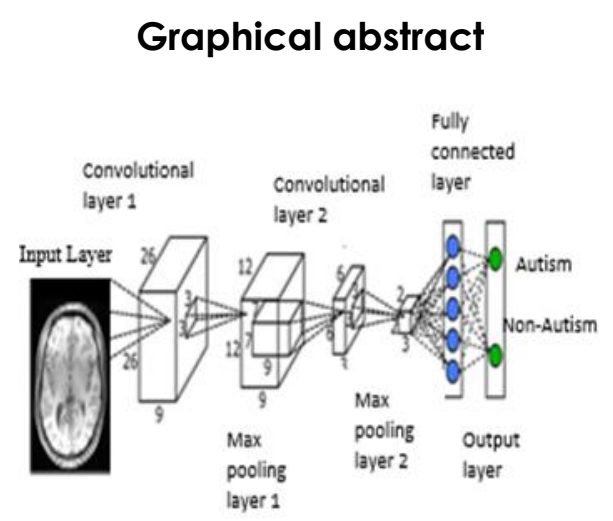

laver 1

\begin{abstract}
Autism Spectrum Disorders (ASDs) define as a scope of disability in the development of certain conditions such as social communication, imagination, and patients' capabilities to make some connection. In Malaysia, the number of ASD cases diagnosed is increasing each year. Typically, ASD patients are analyzed by doctors based on history and behavior observation without the ability to diagnose instantaneously. This research intends to study the ASD biomarker based on neuroimaging functional Magnetic Resonance Imaging (fMRI) images, which can aid doctors in diagnosing ASD. This study applies a deep learning method from Convolutional Neural Network (CNN) variants to detect either the patients are ASD or non-ASD and extract the robust characteristics from neuroimages in fMRI. Then, it interprets the performance of pre-processed images in the form of accuracy to classify the neural patterns. The Autism Brain Imaging Data Exchange (ABIDE) dataset was used to research the brain imaging of ASD patients. The results achieved using CNN models namely VGG-16 and ResNet-50 are $63.4 \%$ and $87.0 \%$ accuracy, respectively. This method also assists doctors in detecting Autism from a quantifiable method that is not dependent on the behavioral observations of suspected autistic children.
\end{abstract}

Keywords: Autism spectrum disorder, biomarker, fMRI, CNN, ABIDE dataset accuracy

\begin{abstract}
Abstrak
Autism Spectrum Disorders (ASD) didefinisikan sebagai ruang pengaluran kecacatan dalam pengembangan keaadaan tertentu seperti komunikasi sosial, imaginasi, dan kemampuan pesakit untuk berinteraksi. Di Malaysia, jumlah kes ASD yang didiagnosis meningkat setiap tahun. Biasanya, pesakit ASD dianalisis oleh doktor berdasarkan sejarah dan pemerhatian tingkah laku tanpa keupayaan untuk menjalankan diagnosis dengan kadar yang segera. Tujuan penyelidikan ini adalah untuk mengkaji biomarker ASD berdasarkan imej functional Magnetic Resonance Imaging (fMRI), yang dapat membantu dalam mendiagnosis ASD. Kajian ini menggunakan kaedah deep learning Convolutional Neural Network (CNN) dengan menggunakan model CNN, VGG-16 dan ResNet50 untuk mengesan pesakit ASD atau bukan ASD dan mengekstrak ciri-ciri kukuh dari neuroimages dalam fMRI. Kemudian, ia menafsirkan prestasi imej yang diproses dan ketepatan CNN dalam mengklasifikasikan corak saraf. Autism Brain Imaging Data Exchange (ABIDE) yang terkenal sebagai pangkalan data
\end{abstract}


pelbagai sisi global digunakan untuk mengkaji pengimejan otak pesakit ASD. Hasil kajian, menggunakan model CNN, VGG-16 dan ResNet-50 masing-masing mencapai $63.4 \%$ dan $87.0 \%$ ketepatan dalam mengesan pesakit ASD berbanding pesakit bukan ASD. Kaedah ini juga dapat membantu doktor dalam melakukan pengesanan prestasi autisme dari kaedah kuantitatif dan tidak hanya bergantung pada pemerhatian tingkah laku kanak-kanak autisme yang disyaki.

Kata kunci: Gangguan spektrum autisme, biomarker, fMRI, $C N N$, set data ABIDE, ketepatan

(C) 2021 Penerbit UTM Press. All rights reserved

\subsection{INTRODUCTION}

In this new era, the number of autisms born is increasing globally. The ratio of Autism recorded with Autism is 1 in 68 children, and boys have the highest risk to be diagnosed with Autism with a prevalence of 4 boys to 1 girl [1]. Autism is a disorder associated with neurodevelopmental that affects the development of the brain. The disease can be determined starting from 16 months of childhood age [5]. However, Autism is not easily detected due to non-existing physical features in autistic children. In a normal approach, the doctors will use a screening tool for early childhood to determine Autism in children from the range of 16 to 30 months of age [23].

Lack of experience and training by the doctors' have caused inaccurate diagnosis of the children with ASD [4]. These problems can deter pediatric screening since a simple approach does not exist in diagnosing ASD. The diagnosis process requires frequent follow-up treatment from the ASD patient individually to acquire an accurate result. However, the doctor can still produce a wrong diagnosis of ASD functional levels since the evaluation is based on subjective observation. Currently, researchers in the related field are focusing on quantifiable methods such as functional Magnetic Resonance Imaging (fMRI) that has been identified as one of the prominent solutions in finding the ASD biomarker [28].

Detecting ASD from complex $f M R I$ images is the challenge in this research. The most possible solution to accurately detect ASD is by using the machine learning (ML) methods such as Support Vector Machine (SVM), Neural Networks, K-nearest neighbor, etc [24-25]. Abraham et al. (2017) proposed a SVC machine learning approach using resting-state fMRI from ABIDE dataset and achieved $67 \%$ of accuracy. On the other hand, Monté-Rubio et al. (2018) worked on MRI images from ABIDE dataset by applying SVM algorithm with accuracy of $62 \%$. However, these approaches are handcrafted types of feature extractors that have limitations in generalizing new image samples. These approaches also have drawbacks in handling data-driven images and big data [26].
Therefore, the state-of-the-art approach in handling complex images like $f M R I$ is the deep learning approach. It has achieved superior accuracy and generalization capabilities in classification, detection, and segmentation [27]. Deep learning is the best method to overcome the aforementioned problems since it is well-known for its robust properties. It can detect images to simplify the problem at hand and can extract robust characteristics of ASD brain images.

One of the most popular deep learning methods is Convolutional Neural Network (CNN). It was used to classify various domain such as heartbeats [18], segmenting the brain regions in MRI [19], face recognition [13], racing bib number recognition [14], license plate recognition [16], object detection systems for blind people [17], etc. CNN was also used to develop an $\mathrm{fMRI-data}$ model to differentiate between ASD and non-ASD patients using the Autism Brain Imaging Data Exchange dataset to create the diagnosis model. Prominent characteristics were extracted and analyzed to detect ASD [4],[6],[21-23]. Z. Sherkatghanad et al. (2019) reported that, an accuracy of $70.22 \%$ achieved by implementing CNN model for ABIDE I dataset. Heinsfield et al. (2018), Dvornek et al. (2018), and Shahamat et al. (2020) uses CNN algorithm to classify ASD using resting-state fMRI data from ABIDE dataset with accuracy of $70 \%$.

This research focuses to design a deep learning model, specifically Convolutional Neural Network (CNN), using fMRI data for detecting the ASD biomarker and evaluate the detection performance in terms of accuracy.

This paper is structured as follows: The proposed methods and the database used are described in the following section. The results and analysis are shown in Section 3. Finally, Section 4 concludes this paper.

\subsection{METHODOLOGY}

This section discusses the methodology of image detection technique for ASD using the fMRI images from the ABIDE dataset. There are five main stages in image detection for Autism namely data-collection, pre-processing, model designing, training, and 
detection. For the designing stage, two CNN models were evaluated in this research, which were VGG-16 [9] and ResNet-50 [10].

The samples were separated into two folders which were the training file and test file. The training file consists of images that were used in the training phase of the CNN model. Meanwhile, the images of the test file were used to evaluate the CNN model performance by measuring the accuracy.

This research aims to come out with the best CNN model in performing ASD detection based on FMRI images. Therefore, detailed information regarding the research is discussed in the following section. The information such as the operating system, machine learning platform, language, types of classification, performance measure, and data sample sizes are shown in Table 1. Figure 1 shows the flowchart of methodology process.

Table 1 List of packages and tools used

\begin{tabular}{ll}
\hline Type of packages & Tools name \\
\hline Operating System & Ubuntu, Linux \\
Datasets Framework & TensorFlow \\
Languages & Python 3 \\
Types of Classification & ASD and Non-ASD \\
Performance Measure & Diagnosis accuracy, \\
& biomarkers detection, fMRI \\
Dataset & ABIDE I (1112 total \\
& samples) and ABIDE II \\
& (1114 total samples) \\
& Convolutional Neural \\
Method & Network (CNN): VGG-16 \\
& and ResNet-50 \\
\hline
\end{tabular}

\subsection{Flowchart of Methodology}

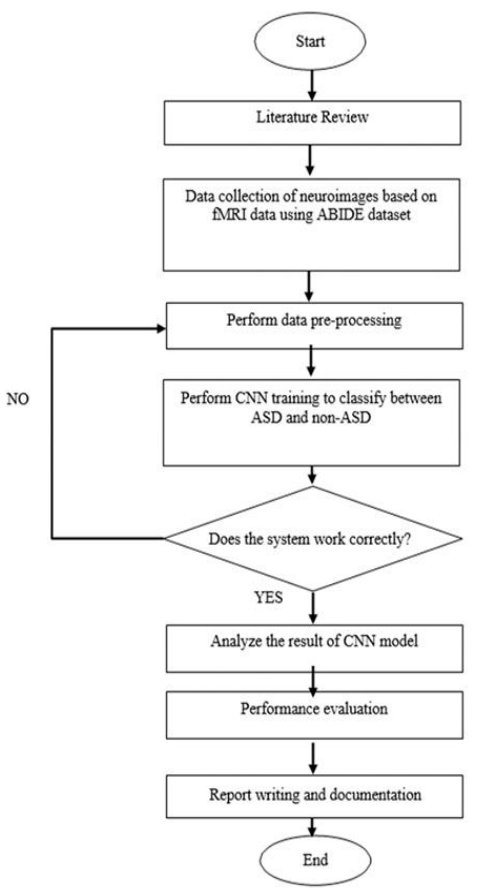

Figure 1 Methodology flowchart process

\subsection{Dataset}

The dataset used in this research is the ABIDE I and ABIDE II datasets. ABIDE I dataset consists of 1112 total samples including 539 patients and 573 typical controls (TC) subjects. While ABIDE II dataset consists of 1114 total samples with 521 patients and 573 typical controls (TC) subjects. The age range for both datasets is between 5-64years. The fMRI images were divided into two folders, which are meant for training and testing samples. There were two other subfolders in each main folder and labeled as Autism and NonAutism.

\subsection{Data Conversion}

The original format of the image dataset is NIfTI-1 data format file (NII format) which is in 3-Dimensional (3D) form. MRIcron software was used to convert the 3D data format into 2D data images. Then, the images can directly be saved in PNG format. Once the images are saved, the PNG format needs to be converted into JPG format using an online data source since TensorFlow can only read JPG format images [8].

\subsection{Data Preprocessing}

In the pre-processing step, the raw data in JPG format are resized to $224 \times 224$ pixels. Then, batch normalization was applied to standardize the inputs to a layer for each mini-batch. This has the effect of stabilizing the learning process and dramatically reducing the number of training periods necessary for deep networks to be trained.

\subsection{Convolutional Neural Network (CNN)}

CNN is extensively used in the area of computer vision. It is a state-of-the-art method to classify sample images, detect objects, and segment images. It consists of several main layers namely the convolutional layer, pooling, and fully connected layers. The function of the convolutional layer is to extract the features from an input. Meanwhile, the pooling layer reduces the parameters of an image if the image size is out of bound or too large. Next, the feature maps are flattened into vectors in a fully connected layer and combined with all the features to create a model. In this research, two pre-trained CNN models were chosen namely VGG-16 and ResNet-50 model.

VGG-16 model has 16 layers. The input image dimension of this network is $(224,224,3)$. The kernel size is $(3 \times 3)$ with 64 channels and the same padding is applied in the first two layers. After the max-pooling layer with stride $(2,2)$, there are two layers with convolution layers of filter sizes $(3,3,256)$ followed by the max-pooling layer. The max-pooling layer provides the function to reduce the volume size. There are about 4096 nodes used in a fully connected layer. 
ResNet-50 architecture model has 50 layers and five stages, and each of the stages consists of convolution and Identity block. Each convolution block has 3 convolution layers and there are also 3 convolution layers in each identity block. There are over 23 million trainable parameters in ResNet-50.

Both of the models will be used to classify between ASD and non-ASD using the ABIDE I and ABIDE II samples.

\subsection{Performance Evaluation}

The performance of the CNN pre-trained model was evaluated using several measurements such as recall, precision, and accuracy. The equations are shown below:

$$
\begin{aligned}
& \text { Accuracy }=(T P+T N) /(T P+T N+F P+F N) \times 100 \% \\
& \text { Recall }=T P /(T P+F N) \times 100 \% \\
& \text { Precision }=T P /(T P+F P) \times 100 \%
\end{aligned}
$$

where TP signifies a true positive, TN signifies a true negative, FP signifies a false positive, and FN signifies a false negative.

\subsection{RESULTS AND DISCUSSION}

\subsection{Raw Data}

The raw data from ABIDE I and ABIDE II datasets were in 3D form. Hence, the datasets were converted into 2D images by using MRIcron software. There are three parts of the brain plane, namely sagittal, axial, and coronal. In this research, the axial plane is used as the input samples. Figure 2 (a) shows the whole three-parts of the brain plane while Figure 2 (b) shows the brain's axial plane.
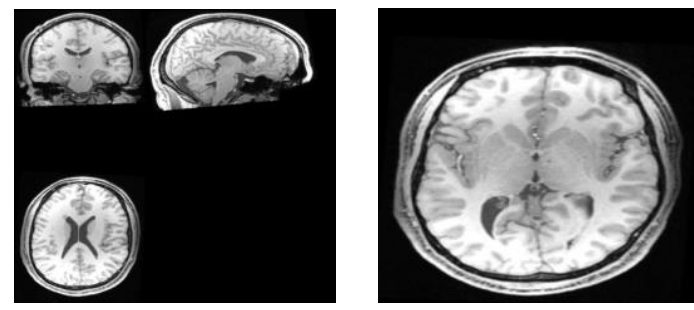

Figure 2 (a): Three planes of the brain (sagittal, axial, and coronal), (b): Axial plane of the brain

\subsection{Pre-processing Image}

After 3D to the 2D conversion of the dataset, the brain images were saved into PNG format and restored to JPG format. The brain images were resized to $224 \times 224$ pixels. Figures 3 and Figure 4 illustrate the brain images before and after prepreprocessing, respectively.

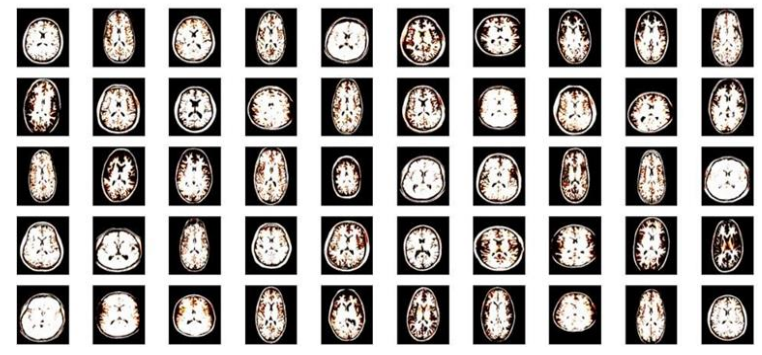

Figure 3 Pre-processing image for Autism Classification: Non-Autism

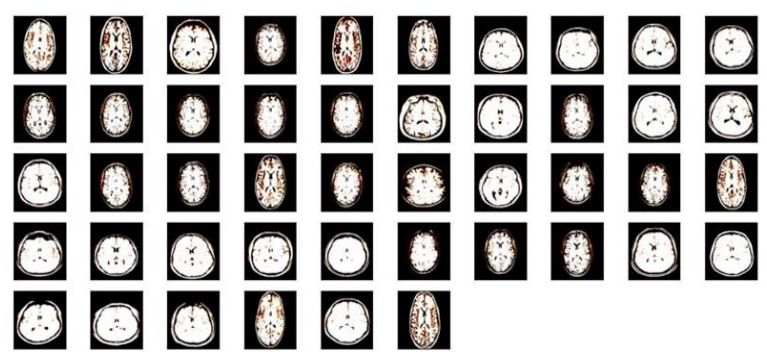

Figure 4 Pre-processing image for Non-Autism

\subsection{Training Process}

Every CNN pre-trained model was trained using the fMRI data from ABIDE I and ABIDE || datasets. The accuracy and losses for training and test set for each model are plotted. The trained weights for both models were saved in a .hfd5 format file and used in the Graphical User Interface (GUI) to evaluate each model's performance.

\subsubsection{VGG-16 Models}

Figure 5 illustrates the summary of VGG-16 model and the training accuracy of the VGG-16 model that increases from $71.0 \%$ to $80.3 \%$. The training accuracy achieves $80 \%$ as the number of batch sizes for training increases. However, validation accuracy is vice-versa with training accuracy. From one to six epochs, the validation accuracy maintains at $73.0 \%$ but suddenly drops at seven and keeps going down until 13. Fortunately, the validation accuracy increases instantly to achieve $70.0 \%$ and dropping again at epoch 15, in which the value is $46.4 \%$. The graph trend is plotted frequently for validation accuracy. Figure 6 and Figure 7 show the graph of train and test accuracy for VGG-16, respectively. 


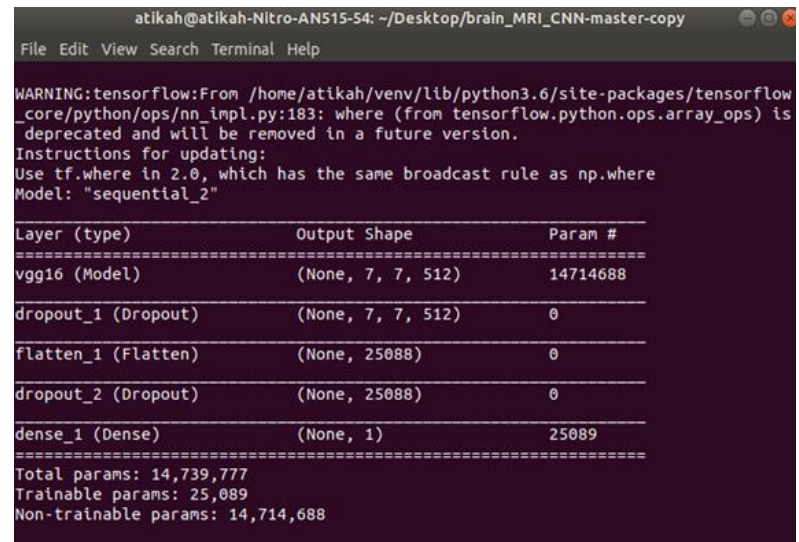

Figure 5 The sequential layer of VGG-16 architecture

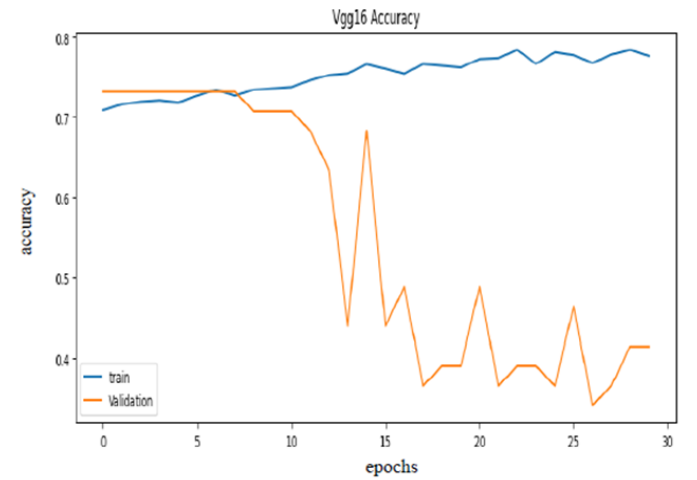

Figure 6 Train versus test accuracy of VGG-16 model

Figure 3.6 shows that the VGG-16 model's training loss is decreasing from $61.5 \%$ to $54.1 \%$. The pattern of the graph continues to go downward. However, the validation loss is going upward from $54 \%$ to more than $100 \%$. This is possibly due to the misdetection of the dataset and affected the validation accuracy. Figure 3.6 portrays the graph of train versus test loss of the VGG-16.

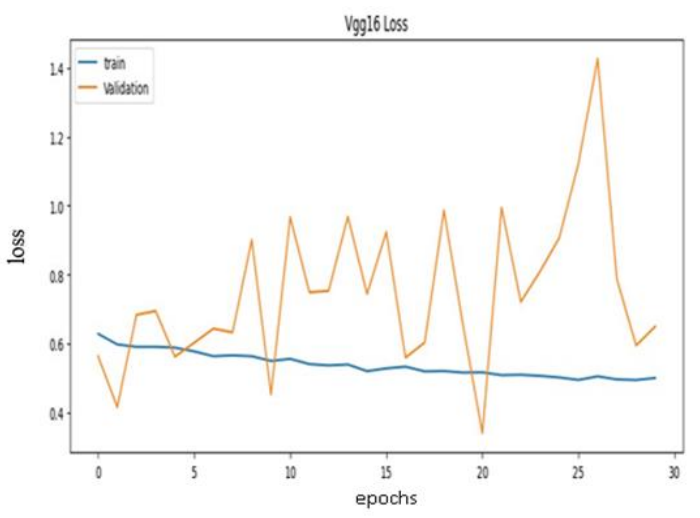

Figure 7 Train versus test loss of VGG-16 model

\subsubsection{ResNet-50 Model}

The ResNet-50 model architecture, short for the Residual Network, is a deep feed-forward neural network with 50 convolutional layers. The figure below illustrates the summary of the ResNet-50 model of a structure. Figure 8 shows the sequential layer of ResNet-50 architecture.

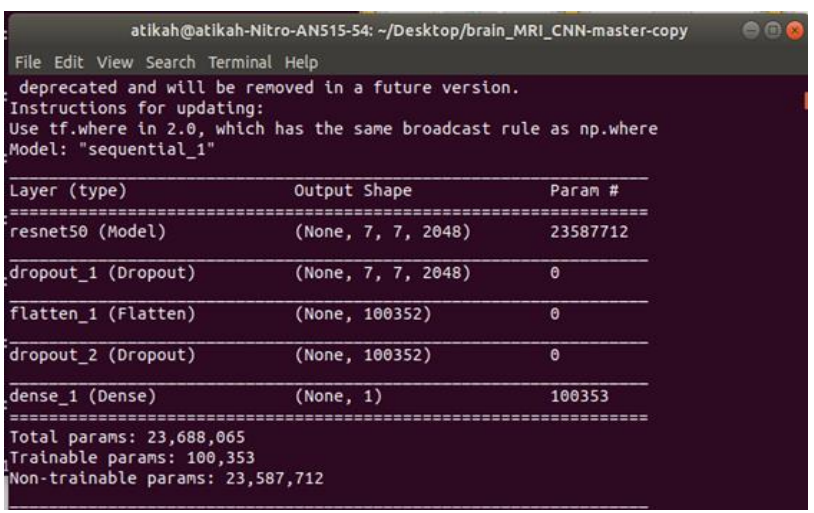

Figure $\mathbf{8}$ The sequential layer of ResNet-50 architecture

The ResNet50 model's training accuracy illustrated in Figure 9 shows an increasing pattern starts from $71.1 \%$ to $98.5 \%$. Meanwhile, the test accuracy for the ResNet50 model rises drastically from 0 epoch to 4 epochs with accuracy from $50.4 \%$ to $85.1 \%$. Then, the test accuracy keeps increasing more than the previous until $89.5 \%$ at epoch 30 . The results show that the ResNet50 model managed to achieve more than $80 \%$ in detecting autism patients.

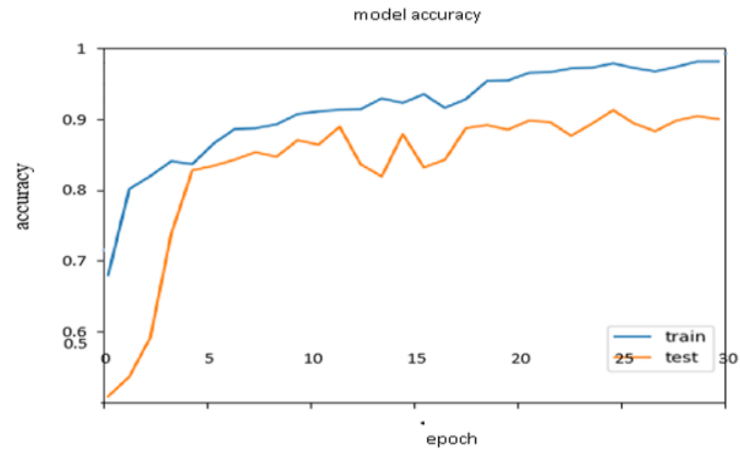

Figure 9 Train versus test accuracy of ResNet-50 model 


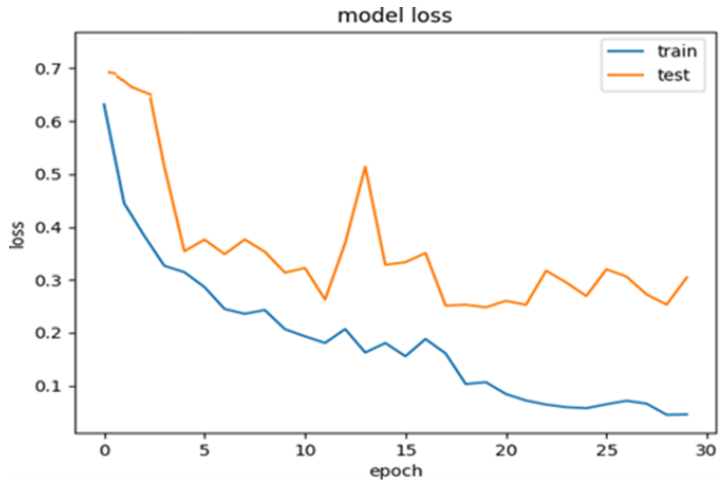

Figure 10 Train versus test loss of ResNet-50 model

Conversely, Figure 10 shows that the train and test loss graph patterns are decreasing. The training loss decreases from $63.4 \%$ to $3 \%$ starting from epoch 0 until epoch 30. The test loss also decreases starting from $72.3 \%$ and suddenly going down steeper at epoch 5 until it reaches 39.2\%. The test loss continues to decrease until epoch 9. However, at epoch 10, the test loss increases from $28.9 \%$ to $51.2 \%$. However, it does not remain too long as at epoch 14 , and the test loss started to decrease until it reaches $31.5 \%$ at epoch 30.

\subsection{Confusion Matrix of VGG-16}

Figure 11 illustrates the confusion matrix of Autism detection using the VGG-16 model, and Table 3.1 shows the classification result based on the feature maps in VGG-16.

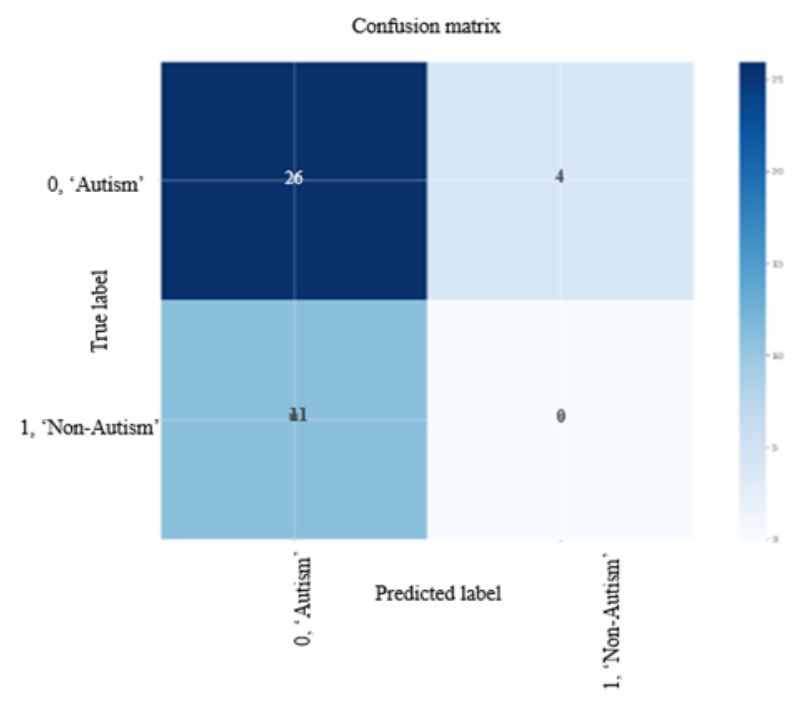

Figure 11 Confusion matrix of Autism for VGG-16

Table 2 The performances of VGG-16

\begin{tabular}{ccc}
\hline Recall & Precision & Accuracy \\
\hline Recall $=\frac{26}{26+11}$ & Precision $=\frac{26}{26+4}$ & Accuracy $=\frac{26}{26+11+4}$ \\
$=0.703 * 100$ & $=0.867 * 100$ & $=0.634 * 100$ \\
$=70.3 \%$ & $=86.7 \%$ & $=63.4 \%$ \\
\hline
\end{tabular}

\subsection{Confusion Matrix of ResNet-50}

ResNet-50 CNN model has 50 layers deep. A trained network loaded from images in this project dataset. The implementation of the ResNet50 model architecture is done manually and saved in the new model weight. Figure 12 illustrates the confusion matrix of autism detection for the ResNet-50 model, and Table 3 shows the result of the ResNet50 CNN model.

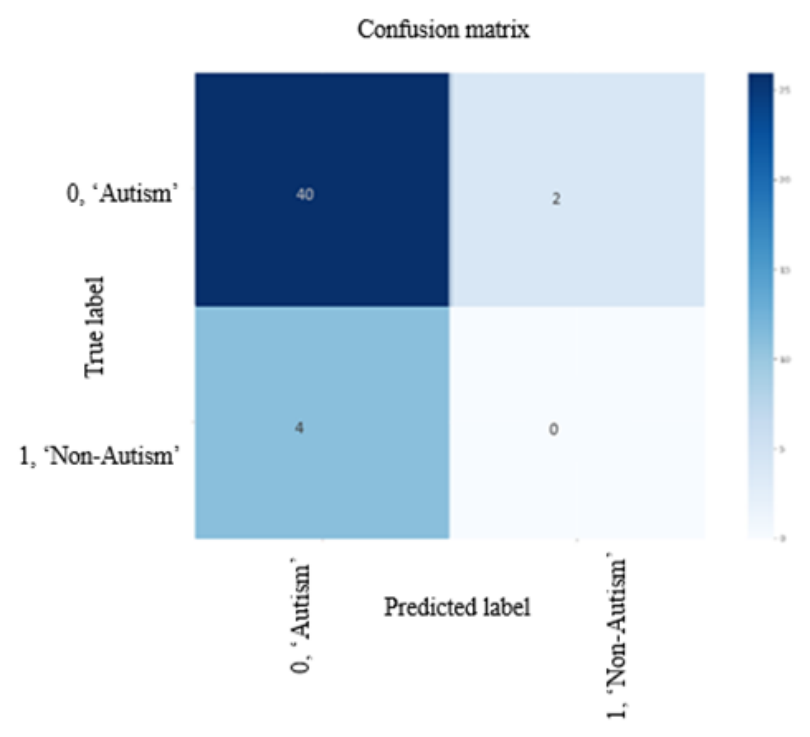

Figure 12 Confusion matrix of Autism detection for ResNet50 model

Table 3 The performance of ResNet50

\begin{tabular}{|c|c|c|}
\hline Recall & Precision & Accuracy \\
\hline Recall $=40$ & Precision $=\frac{40}{2}$ & 40 \\
\hline $\begin{aligned} & 40+4 \\
= & 0.909 * 100\end{aligned}$ & $\begin{aligned} & 40+2 \\
= & 0.952 * 100\end{aligned}$ & $\begin{aligned} & 40+4+2 \\
= & 0.87 * 100\end{aligned}$ \\
\hline$=90.9 \%$ & $=95.2 \%$ & $=87.0 \%$ \\
\hline
\end{tabular}

Table 2 and Table 3 shows the equation used to find the training and test accuracy. ResNet50 model architecture has shown the best performance in detecting Autism compared to VGG-16 model with an accuracy of $87.0 \%$.

A GUI was designed to demonstrate the classification result. The GUI was built to ease users' understanding of the ASD detection result. Figure 13 shows the GUI representation for the brain classification of ASD patients. In the GUI, the user needs to upload an image file from a folder and the user needs to select the type of pre-trained CNN model for classification. The detail of the brain image is represented on the .csv file. When the classify button is pressed, the GUI will show the result obtain based on the model selected. 


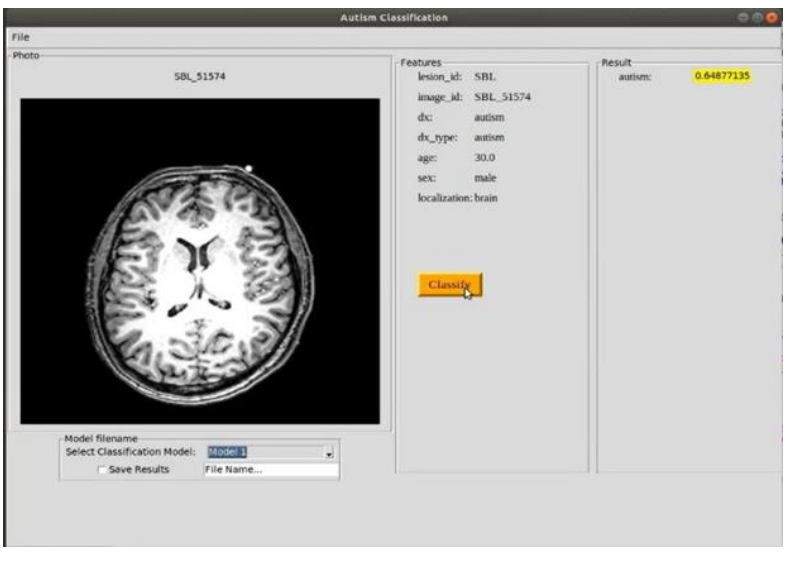

Figure 13 Graphical User Interface (GUI)

\subsection{CONCLUSION}

In conclusion, the aim of this research to detect ASD based on functional Magnetic Resonance Imaging (fMRI) images has achieved, which can aid doctors in diagnosing ASD. CNN pre-trained models namely VGG-16 and Resnet50 were used to detect autism patients. The performance of this system was measured through the accuracy obtained from the result of training the CNN models. A GUI was also built to ease the users in conducting the system.

The performance measures that have been used to evaluate the CNN pre-trained model were recall, precision, and accuracy. The main factors that can affect the accuracy of the system are the number of layers, feature maps, and the size of the sample data. The results achieved using CNN models, VGG16 and ResNet-50 were $63.4 \%$ and $87.0 \%$ accuracy, respectively. The best $\mathrm{CNN}$ model in this project was achieved by ResNet50, with an accuracy of $87.0 \%$.

\section{Acknowledgement}

The authors would like to thank Universiti Teknikal Malaysia Melaka (UTeM), Machine Learning and Signal Processing (MLSP) research group under Centre for Telecommunication Research \& Innovation (CeTRI), and Ministry of Higher Education (MOHE), Malaysia, for sponsoring this work under project FRGS/2018/FKEKK-CERIA/F00363 and the use of the existing facilities to complete this project.

\section{References}

[1] U. Lahiri, K. C. Welch, Z. Warren, and N. Sarkar. 2011. Understanding Psychophysiological Response to a Virtual Reality-based Social Communication System for Children with ASD. 2011 International Conference on Virtual Rehabilitation, ICVR 2011.

[2] M. Sharda et al. 2017. Language Ability Predicts Cortical Structure and Covariance in Boys with Autism Spectrum Disorder. Cereb. Cortex. 27(3): 1849-1862.
[3] J. Jennings Dunlap. 2019. Autism Spectrum Disorder Screening and Early Action. J. Nurse Pract. 15(7): 496-501.

[4] Manaswi, N. K., \& John, S. 2018. Deep Learning with Applications Using Python Berkeley. CA: Apress. 171-197.

[5] Nur, A. A. 2020. Autism Spectrum Disorder Classification on Electroencephalogram Signal Using Deep Learning Algorithm. IAES International Journal of Artificial Intelligence. 9(1): 91.

[6] Sherkatghanad, Z., Akhondzadeh, M., Salari, S., ZomorodiMoghadam, M., Abdar, M., Acharya, U. R., Khosrowabadi, R., \& Salari, V. 2020. Automated Detection of Autism Spectrum Disorder Using a Convolutional Neural Network. Frontiers in Neuroscience. 13. https://doi.org/10.3389/fnins.2019.01325

[7] Huettel, S. A., Song, A. W., \& McCarthy, G. 2014. Functional Magnetic Resonance Imaging (Vol. 3). Sunderland, MA: Sinauer Associates.

[8] Ceder, N. R., McDonald, K., \& Harms, D. D. 2010. The Quick Python Book (Vol. 2). Manning.

[9] Zhang, X., Zou, J., He, K., \& Sun, J. 2015. Accelerating Very Deep Convolutional Networks for Classification and Detection. IEEE Transactions on Pattern Analysis and Machine Intelligence. 38(10): 1943-1955.

[10] Wen, L., Li, X., \& Gao, L. 2019. A Transfer Convolutional Neural Network for Fault Diagnosis based on ResNet-50. Neural Computing and Applications. 1-14.

[11] B. Tang et al. 2016. Deepchart: Combining Deep Convolutional Networks and Deep Belief Networks in Chart Classification. Signal Processing. 124: 156-161.

[12] N. A. Ali, A. R. Syafeeza, L. J. Geok, Y. C. Wong, N. A. Hamid, And A. S. Jaafar. 2019. Design of Automated Computer-aided Classification of Brain Tumor Using Deep Learning. Intelligent and Interactive Computing. 285-291.

[13] Ahmad Radzi, S. 2016. A MATLAB-based Convolutional Neural Network Approach for Face Recognition System. Journal of Bioinformatics and Proteomics Review. 2: 1-5.

[14] Wong, Y. C., Choi, L. J., Singh, R. S. S., Zhang, H., \& Syafeeza, A. R. 2019. Deep Learning-based Racing Bib Number Detection and Recognition. Jordanian Journal of Computers and Information Technology (JJCIT). 5(03).

[15] Marzuki, P., Syafeeza, A. R., Wong, Y. C., Hamid, N. A., Alisa, A. N., \& Ibrahim, M. M. 2019. A Design of License Plate Recognition System Using Convolutional Neural Network. International Journal of Electrical \& Computer Engineering. 9(3): 2088-8708.

[16] Wong, Y. C., Lai, J. A., Ranjit, S. S. S., Syafeeza, A. R., \& Hamid, N. A. 2019. Convolutional Neural Network for Object Detection System for Blind People. Journal of Telecommunication, Electronic and Computer Engineering (JTEC). 11 (2): 1-6.

[17] Pham, T. C., Luong, C. M., Visani, M., \& Hoang, V. D. 2018. Deep CNN and Data Augmentation for Skin Lesion Classification. Asian Conference on Intelligent Information and Database Systems. Springer, Cham. 573-582.

[18] Acharya, U. R., Oh, S. L., Hagiwara, Y., Tan, J. H., Adam, M., Gertych, A., \& San Tan, R. 2017. A Deep Convolutional Neural Network Model to Classify Heartbeats. Computers in Biology and Medicine. 89: 389-396.

[19] Milletari, F., Ahmadi, S. A., Kroll, C., Plate, A., Rozanski, V. Maiostre, J., ... \& Navab, N. 2017. Hough-CNN: Deep Learning for Segmentation of Deep Brain Regions in MRI and Ultrasound. Computer Vision and Image Understanding. 164: 92-102.

[20] LeCun, Y., Bengio, Y., \& Hinton, G. 2015. Deep Learning. Nature. 521 (7553): 436-444.

[21] Shahamat, H., \& Saniee Abadeh, M. 2020. Brain MRI Analysis Using a Deep Learning Based Evolutionary Approach. Neural Networks. 126: 218-234. https://doi.org/10.1016/j.neunet.2020.03.017.

[22] Dvornek, N. C., Ventola, P., \& Duncan, J. S. 2018. Combining Phenotypic and Resting-State Fmri Data for Autism Classification with Recurrent Neural Networks. Proceedings - International Symposium on Biomedical 
Imaging. 2018-April (Isbi): $\quad$ 725-728. https://doi.org/10.1109/ISBI.2018.8363676.

[23] Heinsfeld, A. S., Franco, A. R., Craddock, R. C., Buchweitz, A., \& Meneguzzi, F. 2018. Identification of Autism Spectrum Disorder Using Deep Learning and the ABIDE Dataset. Neurolmage: Clinical.

https://doi.org/10.1016/j.nicl.2017.08.017.

[24] Abraham, A., Milham, M. P., Di Martino, A., Craddock, R. C., Samaras, D., Thirion, B., \& Varoquaux, G. 2017. Deriving Reproducible Biomarkers from Multi-site Resting-state Data: An Autism-based Example. Neurolmage. 147(November 2016): 736-745. https://doi.org/10.1016/j.neuroimage.2016.10.045.

[25] Monté-Rubio, G. C., Falcón, C., Pomarol-Clotet, E., \& Ashburner, J. 2018. A comparison of Various MRI Feature Types for Characterizing Whole Brain Anatomical
Differences Using Linear Pattern Recognition Methods. Neurolmage. 178(April): 753-768. https://doi.org/10.1016/j.neuroimage.2018.05.065.

[26] Koirala, A., Walsh, K. B., Wang, Z., \& McCarthy, C. 2019. Deep learning-Method Overview and Review of Use for Fruit Detection and Yield Estimation. Computers and Electronics in Agriculture. 162: 219-234.

[27] Wen, D., Wei, Z., Zhou, Y., Li, G., Zhang, X. and Han, W., 2018. Deep Learning Methods to Process Fmri Data and Their Application in the Diagnosis of Cognitive Impairment: A Brief Overview and Our Opinion. Frontiers in Neuroinformatics. 12: 23.

[28] Klin, A. 2018. Biomarkers in Autism Spectrum Disorder: Challenges, Advances, and the Need for Biomarkers of Relevance to Public Health. Focus. 16(2): 135-142. 